Pacific

Journal of

Mathematics

UPPER BOUNDS FOR THE FIRST EIGENVALUE OF THE LAPLACIAN ON COMPACT SUBMANIFOLDS

Jean-François Grosjean

Volume $206 \quad$ No. 1

September 2002 


\title{
UPPER BOUNDS FOR THE FIRST EIGENVALUE OF THE LAPLACIAN ON COMPACT SUBMANIFOLDS
}

\author{
JEAN-FranÇOIS GRosJeAn
}

\begin{abstract}
Let $\left(M^{m}, g\right)$ be a compact Riemannian manifold isometrically immersed in a simply connected space form (euclidean space, sphere or hyperbolic space). The purpose of this paper is to give optimal upper bounds for the first nonzero eigenvalue of the Laplacian of $\left(M^{m}, g\right)$ in terms of $r$-th mean curvatures and scalar curvature. As consequences, we obtain some rigidity results. In particular, we prove that if $\left(M^{n}, g\right)$ is a compact hypersurface of positive scalar curvature immersed in $\mathbb{R}^{n+1}$ and if $g$ is a Yamabe metric, then $\left(M^{n}, g\right)$ is a standard sphere.
\end{abstract}

\section{Introduction.}

Let $\left(M^{m}, g\right)$ be a compact, connected $m$-dimensional Riemannian manifold without boundary isometrically immersed into a simply connected space form $N^{n}(\kappa)(\kappa=0,1$ or -1 respectively for Euclidean space, sphere or hyperbolic space) whose canonical metric will be denoted by $h$. A well-known inequality gives an extrinsic upper bound for the first nonzero eigenvalue $\lambda_{1}(M)$ of the Laplacian of $\left(M^{m}, g\right)$ in terms of the square of the length of the mean curvature, denoted by $|H|^{2}$. Indeed, we have

$$
\lambda_{1}(M) V(M) \leq m \int_{M}\left(|H|^{2}+\kappa\right) d v_{g}
$$

where $d v_{g}$ and $V(M)$ denote respectively the Riemannian volume element and the volume of $\left(M^{m}, g\right)$. Moreover the equality holds if and only if $\left(M^{m}, g\right)$ is minimally immersed in a geodesic sphere of $N^{n}(\kappa)$. For $\kappa=0$, this inequality was proved by Reilly $([\mathbf{1 6}])$ and can easily be extended to the spherical case $\kappa=1$ by considering the canonical embedding of $\mathbb{S}^{n}$ in $\mathbb{R}^{n+1}$ and by applying the inequality (1) for $\kappa=0$ to the obtained immersion of $\left(M^{m}, g\right)$ in $\mathbb{R}^{n+1}$. For immersions of $\left(M^{m}, g\right)$ in the hyperbolic space, Heintze ([10]) first proved an $L_{\infty}$ equivalent of (1) and conjectured (1) which was finally obtained by El Soufi and Ilias in [7]. In [16], Reilly has shown estimates of the $\lambda_{1}(M)$ of orientable manifolds $\left(M^{m}, g\right)$ isometrically immersed in $\mathbb{R}^{n}$ in terms of more general invariants called $r$-th mean 
curvatures. Let us first define these invariants. Let $B$ be the second fundamental form of the immersion, which is normal-vector valued, and let $\left(B_{i j}\right)$, be its matrix with respect to an orthonormal frame $\left(e_{i}\right)_{1 \leq i \leq m}$ at a point $x$ of $\left(M^{m}, g\right)$. For any integer $r \in\{1, \ldots, m\}$, the $r$-th mean curvature of the immersion is the quantity, if $r$ is even

$$
H_{r}=\left(\begin{array}{c}
m \\
r
\end{array}\right)^{-1} \frac{1}{r !} \sum_{\substack{i_{1} \ldots i_{r} \\
j_{1} \ldots j_{r}}} \epsilon\left(\begin{array}{c}
i_{1} \ldots i_{r} \\
j_{1} \ldots j_{r}
\end{array}\right) h\left(B_{i_{1} j_{1}}, B_{i_{2} j_{2}}\right) \ldots h\left(B_{i_{r-1} j_{r-1}}, B_{i_{r} j_{r}}\right)
$$

and if $r$ is odd

$$
\begin{aligned}
H_{r}=\left(\begin{array}{c}
m \\
r
\end{array}\right)^{-1} \frac{1}{r !} \sum_{\substack{i_{1} \ldots i_{r} \\
j_{1} \ldots j_{r}}} \epsilon\left(\begin{array}{c}
i_{1} \ldots i_{r} \\
j_{1} \ldots j_{r}
\end{array}\right) \\
\cdot h\left(B_{i_{1} j_{1}}, B_{i_{2} j_{2}}\right) \ldots h\left(B_{i_{r-2} j_{r-2}}, B_{i_{r-1} j_{r-1}}\right) B_{i_{r} j_{r}}
\end{aligned}
$$

where $\epsilon\left(\begin{array}{c}i_{1} \ldots i_{r} \\ j_{1} \ldots j_{r}\end{array}\right)$ is zero if $\left\{i_{1}, \ldots, i_{r}\right\} \neq\left\{j_{1}, \ldots, j_{r}\right\}$ or if there exists $p$ and $q$ such that $i_{p}=i_{q}$, and in the contrary case $\epsilon\left(\begin{array}{c}i_{1} \ldots i_{r} \\ j_{1} \ldots j_{r}\end{array}\right)$ is the signature of the permutation of $\left(\begin{array}{c}i_{1} \ldots i_{r} \\ j_{1} \ldots j_{r}\end{array}\right)$. By convention, we put $H_{0}=1$ and $H_{m+1}=0$. Note that $H_{1}$ is nothing but the usual mean curvature vector and for submanifolds of $\mathbb{R}^{n}, H_{2}$ is up to a multiplicative coefficient the scalar curvature. If the codimension is 1 and if $\left(M^{m}, g\right)$ is oriented by a normal vector field $\nu$, it is convenient to work with the real valued second fundamental form $b$ by: $b(X, Y)=h(B(X, Y), \nu)$. Therefore, the $r$-th mean curvatures of odd order can be defined as real valued (we replace in this case the vector field $H_{r}$ by the scalar $h\left(H_{r}, \nu\right)$ ). Choosing an orthonormal frame at $x$ such that $b_{x}\left(e_{i}, e_{j}\right)=\mu_{i} \delta_{i j}$, we get the following unified formulae, for any integer $r \in\{1, \ldots, m\}$

$$
H_{r}=\left(\begin{array}{c}
m \\
r
\end{array}\right)^{-1} \sum_{i_{1}<\cdots<i_{r}} \mu_{i_{1}} \ldots \mu_{i_{r}}
$$

In [16], Reilly proved a sharp bound for $\lambda_{1}(M)$ of manifolds immersed in a Euclidean space, in terms of $r$-th mean curvatures. Recall this result:

Theorem 1.1 (see Reilly [16], Theorem A). Let $\left(M^{m}, g\right)$ be a compact, orientable m-dimensional Riemannian manifold isometrically immersed by $\phi$ into $\mathbb{R}^{n}$. 
1. If $m<n-1$ and if $r$ is an even integer such that $r \in\{0, \ldots, m-1\}$, then

$$
\lambda_{1}(M)\left(\int_{M} H_{r} d v_{g}\right)^{2} \leq m V(M) \int_{M}\left|H_{r+1}\right|^{2} d v_{g} .
$$

Moreover if $H_{r+1}$ doesn't vanish identically and if equality holds, then $\phi$ immerses $\left(M^{m}, g\right)$ minimally into some hypersphere in $\mathbb{R}^{n}$.

2. If $m=n-1$ and $r \in\{0, \ldots, m-1\}$, then

$$
\lambda_{1}(M)\left(\int_{M} H_{r} d v_{g}\right)^{2} \leq m V(M) \int_{M} H_{r+1}^{2} d v_{g} .
$$

Moreover if $H_{r+1}$ doesn't vanish identically, equality holds if and only if $\phi$ immerses $\left(M^{m}, g\right)$ as a hypersphere in $\mathbb{R}^{n}$.

Note that, if $m<n-1$ and $r$ is odd, there is no inequality, because in the proof it is necessary that $H_{r}$ can be viewed as a real quantity.

The purpose of this paper is to find similar upper bounds for submanifolds of the other space forms. In a first part, we extend Reilly's result to the sphere and the hyperbolic space (Theorems 2.1 and 2.2). In a second part, as a consequence of such estimates and using a different approach, we obtain for hypersurfaces of a simply connected space form upper bounds of $\lambda_{1}(M)$ in terms of the scalar curvature (Corollary 3.1 and Theorem 3.1). Moreover, these estimates allow us to obtain rigidity results (Remark 3.1). In particular, we prove that if $\left(M^{n}, g\right)$ is a compact hypersurface of positive scalar curvature immersed in the Euclidean space and if $g$ is a Yamabe metric, then $\left(M^{n}, g\right)$ is a standard sphere (Corollary 3.2).

\section{Upper bounds of $\lambda_{1}(M)$ in terms of $r$-th mean curvatures.}

Let $\left(M^{m}, g\right)$ be an orientable $m$-dimensional Riemannian manifold isometrically immersed by $\phi$ in an $n$-dimensional Riemannian manifold $\left(N^{n}, h\right)$ of constant sectional curvature. Let $B$ be the second fundamental form associated to $\phi$. Before stating our results, we need some definitions. Let $\left(e_{i}\right)_{1 \leq i \leq m}$ be an orthonormal frame at $x \in M,\left(e_{i}^{\star}\right)_{1 \leq i \leq m}$ its dual coframe and $\left(B_{i j}\right)$ the matrix of $B$ with respect to the frame $\left(e_{i}\right)_{1 \leq i \leq m}$. We define the following $(0,2)$-tensors $T_{r}$ for $r \in\{1, \ldots, m\}$ :

- If $r$ is even, we set

$$
T_{r}=\frac{1}{r !} \sum_{\substack{i, i_{1} \ldots i_{r} \\
j, j_{1} \ldots j_{r}}} \epsilon\left(\begin{array}{c}
i i_{1} \ldots i_{r} \\
j j_{1} \ldots j_{r}
\end{array}\right) h\left(B_{i_{1} j_{1}}, B_{i_{2} j_{2}}\right) \ldots h\left(B_{i_{r-1} j_{r-1}}, B_{i_{r} j_{r}}\right) e_{i}^{\star} \otimes e_{j}^{\star}
$$


- If $r$ is odd, we set

$$
\begin{array}{r}
T_{r}=\frac{1}{r !} \sum_{\substack{i, i_{1} \ldots i_{r} \\
j, j_{1} \ldots j_{r}}} \epsilon\left(\begin{array}{c}
i i_{1} \ldots i_{r} \\
j j_{1} \ldots j_{r}
\end{array}\right) h\left(B_{i_{1} j_{1}}, B_{i_{2} j_{2}}\right) \ldots \\
h\left(B_{i_{r-2} j_{r-2}}, B_{i_{r-1} j_{r-1}}\right) B_{i_{r} j_{r}} \otimes e_{i}^{\star} \otimes e_{j}^{\star} .
\end{array}
$$

By convention $T_{0}=g$. As for the $r$-th mean curvatures, we have an unified formulae if the codimension of $\left(M^{m}, g\right)$ is 1 (i.e., $\left.m=n-1\right)$; indeed, choosing a unit normal field $\nu$ and a $g$-orthonormal frame $\left(e_{i}\right)_{1 \leq i \leq m}$ at a point $x \in$ $M$ which diagonalizes the scalar valued second fundamental form $b$ (i.e., $\left.b_{x}\left(e_{i}, e_{j}\right)=\mu_{i} \delta_{i j}\right)$, the tensors $T_{r}$ can be viewed as scalar valued $(0,2)$ tensors (if $r$ is odd we replace $T_{r}$ by the tensor $h\left(T_{r}(.,),. \nu\right)$ ) and we have at $x$

$$
T_{r}=\left(\begin{array}{c}
m \\
r
\end{array}\right)^{-1} \sum_{\substack{i_{1}<\cdots<i_{r} \\
i_{j} \neq i}} \mu_{i_{1}} \ldots \mu_{i_{r}} e_{i}^{\star} \otimes e_{i}^{\star} .
$$

We first prove a lemma which is well-known in codimension 1:

Lemma 2.1. Let $\left(M^{m}, g\right)$ be a n-dimensional Riemannian manifold isometrically immersed in a n-dimensional Riemannian manifold of constant sectional curvature. Let $r \in\{1, \ldots, m\}$, and if $m<n-1$, assume that $r$ is even. Then we have

$$
\operatorname{div}_{M} T_{r}=0 .
$$

Proof. The proof is known when $m=n-1$ (see for instance [17]). Assume that $m<n-1$ and $r$ is even and let $\nabla^{M}$ denote the Riemannian connection of $\left(M^{m}, g\right)$. Let $x \in M$ and $\left(e_{i}\right)_{1 \leq i \leq m}$ be an orthonormal parallel frame at $x$, then we have

$$
\begin{aligned}
& \operatorname{div}_{M} T_{r}\left(e_{j}\right) \\
& =\frac{1}{r !} \sum_{i} \nabla_{e_{i}} T_{r}\left(e_{i}, e_{j}\right) \\
& =\frac{1}{(r-1) !} \sum_{\substack{i_{1} \ldots i_{r} \\
j_{1} \ldots j_{r} \\
i}} \epsilon\left(\begin{array}{c}
i i_{1} \ldots i_{r} \\
j j_{1} \ldots j_{r}
\end{array}\right) h\left(\left(\nabla_{e_{i}} B\right)_{i_{1} j_{1}}, B_{i_{2} j_{2}}\right) \ldots h\left(B_{i_{r-1} j_{r-1}}, B_{i_{r} j_{r}}\right) \\
& =\frac{1}{(r-1) !} \sum_{\substack{i_{1} \ldots i_{r} \\
j_{1} \ldots j_{r}}} \epsilon\left(\begin{array}{c}
i i_{1} \ldots i_{r} \\
j j_{1} \ldots j_{r}
\end{array}\right) h\left(\left(\nabla_{e_{i_{1}}} B\right)_{i j_{1}}, B_{i_{2} j_{2}}\right) \ldots h\left(B_{i_{r-1} j_{r-1}}, B_{i_{r} j_{r}}\right)
\end{aligned}
$$


where we used in the last equality the Codazzi equation and the fact that the sectional curvature of $\left(N^{n}, h\right)$ is constant. Therefore

$$
\begin{aligned}
& \operatorname{div}_{M} T_{r}\left(e_{j}\right)= \frac{1}{(r-1) !} \sum_{\substack{i_{1} \ldots i_{r} \\
j_{1} \ldots j_{r}}} \epsilon\left(\begin{array}{c}
i_{1} i \ldots i_{r} \\
j j_{1} \ldots j_{r}
\end{array}\right) h\left(\left(\nabla_{e_{i}} B\right)_{i_{1} j_{1}}, B_{i_{2} j_{2}}\right) \ldots \\
& h\left(B_{i_{r-1} j_{r-1}}, B_{i_{r} j_{r}}\right) \\
&=-\frac{1}{(r-1) !} \sum_{\substack{i_{1} \ldots i_{r} \\
j_{1} \ldots j_{r} \\
i}} \epsilon\left(\begin{array}{c}
i i_{1} \ldots i_{r} \\
j j_{1} \ldots j_{r}
\end{array}\right) h\left(\left(\nabla_{e_{i}} B\right)_{i_{1} j_{1}}, B_{i_{2} j_{2}}\right) \ldots \\
& h\left(B_{i_{r-1} j_{r-1}}, B_{i_{r} j_{r}}\right) \\
&=-\operatorname{div}_{M} T_{r}\left(e_{j}\right) .
\end{aligned}
$$

This completes the proof.

In the following lemma, we give some relations between the $r$-th mean curvatures and the tensors $T_{r}$. These relations are also well-known in codimension 1 (see for instance [17]).

Lemma 2.2. For any integer $r \in\{1, \ldots, m\}$, we have

$$
\operatorname{tr}\left(T_{r}\right)=k(r) H_{r}
$$

Moreover, if $r$ is even

$$
\sum_{i j} T_{r}\left(e_{i}, e_{j}\right) B\left(e_{i}, e_{j}\right)=k(r) H_{r+1}
$$

and if $r$ is odd

$$
\sum_{i j} h\left(T_{r}\left(e_{i}, e_{j}\right), B\left(e_{i}, e_{j}\right)\right)=k(r) H_{r+1}
$$

where $k(r)=(m-r)\left(\begin{array}{c}m \\ r\end{array}\right)$.

Proof. It follows easily from the definitions of $T_{r}$ and $H_{r}$, so we will omit it.

Now, we extend Theorem 1.1 of Reilly mentioned in the introduction to submanifolds of the sphere.

Theorem 2.1. Let $\left(M^{m}, g\right)$ be a compact, orientable $m$-dimensional Riemannian manifold isometrically immersed by $\phi$ into $\mathbb{S}^{n}$. 
1. If $m<n-1$ and if $r$ is an even integer such that $r \in\{0, \ldots, m-1\}$, then

$$
\lambda_{1}(M)\left(\int_{M} H_{r} d v_{g}\right)^{2} \leq m V(M) \int_{M}\left(\left|H_{r+1}\right|^{2}+H_{r}^{2}\right) d v_{g} .
$$

Moreover, if $H_{r}$ doesn't vanish identically, and if equality holds then $\phi$ immerses $M$ minimally into $\mathbb{S}^{n}$ or some geodesic hypersphere of $\mathbb{S}^{n}$.

2. If $m=n-1$ and $r \in\{0, \ldots, m-1\}$, then

$$
\lambda_{1}(M)\left(\int_{M} H_{r} d v_{g}\right)^{2} \leq m V(M) \int_{M}\left(H_{r+1}^{2}+H_{r}^{2}\right) d v_{g} .
$$

If $H_{r}$ doesn't vanish identically and if equality holds, then $\left(M^{m}, g\right)$ is minimally immersed in $\mathbb{S}^{n}$ or $\phi(M)$ is a geodesic sphere. Moreover, if $\phi(M)$ is contained in a hemisphere, we have equality if and only if $\phi$ immerses $\left(M^{m}, g\right)$ as a geodesic hypersphere of $\mathbb{S}^{n}$.

Remark 2.1. As in Theorem 1.1, the method used doesn't allow us to have an inequality if $m<n-1$ and $r$ is odd.

On the other hand, this theorem can't be deduced from Theorem 1.1 of Reilly by considering the canonical embedding of $\mathbb{S}^{n}$ in $\mathbb{R}^{n+1}$, but is a consequence of a more general result given in Proposition 2.1 below.

Let $\left(M^{m}, g\right)$ be a compact $m$-dimensional Riemannian manifold isometrically immersed by $\phi$ in $\mathbb{R}^{n}$ and denotes by $B$ its second fundamental form. We assume that $\left(M^{m}, g\right)$ is endowed with a free divergence $(0,2)$-tensor $T$ and we define a normal vector field $H_{T}$ at a point $x \in M$, by

$$
H_{T}(x)=\sum_{1 \leq i, j \leq n} T\left(e_{i}, e_{j}\right) B\left(e_{i}, e_{j}\right)
$$

where $\left(e_{i}\right)_{1 \leq i \leq m}$ is an orthonormal basis of the tangent space of $M$ at $x$. We have the following generalization of Theorem 1.1:

Proposition 2.1. Let $\left(M^{m}, g\right)$ be a compact, orientable $m$-dimensional Riemannian manifold isometrically immersed by $\phi$ into $\mathbb{R}^{n}$ and assume that $\left(M^{m}, g\right)$ is endowed with a free divergence $(0,2)$-tensor $T$. Then, we have

$$
\lambda_{1}(M)\left(\int_{M} \operatorname{tr}(T) d v_{g}\right)^{2} \leq m V(M)\left(\int_{M}\left|H_{T}\right|^{2} d v_{g}\right) .
$$

Moreover, if $H_{T}$ doesn't vanish identically and if equality holds, then $\left(M^{m}, g\right)$ is minimally immersed into a geodesic hypersphere of $\mathbb{R}^{n}$.

This proposition will be a consequence of a generalization of the HsiungMinkowski formulas. For this purpose, let us first define a second order differential operator $L_{T}$ on $C^{\infty}(M)$ by

$$
L_{T} u=-\operatorname{div}_{M}\left(T^{\sharp} \nabla^{M} u\right)
$$


where $\nabla^{M}$ is the gradient associated to the metric $g$ and $T^{\sharp}$ is the symmetric endomorphism associated to $T$ with respect to $g$ (i.e., $g\left(T^{\sharp} X, Y\right)=$ $T(X, Y))$. The differential operator $L_{T}$ is self-adjoint because $T$ is a freedivergence tensor, and it is easy to see that

$$
L_{T}(u)=-\left\langle D^{2} u, T\right\rangle
$$

where $D^{2}$ and $\langle$,$\rangle denote respectively the hessian operator and the inner$ product extended to tensors. Now, if $\left(\partial_{i}\right)_{1 \leq i \leq n}$ and $\phi^{i}$ denote respectively the canonical basis of $\mathbb{R}^{n}$ and the component functions of $\phi$ in this basis, we set

$$
L_{T} \phi=\sum_{i \leq n} L_{T} \phi^{i} \partial_{i}
$$

Now, we can state:

Lemma 2.3. We have

$$
L_{T} \phi=-H_{T}
$$

and

$$
\frac{1}{2} L_{T}|\phi|^{2}=-\left\langle\phi, H_{T}\right\rangle-\operatorname{tr}(T) .
$$

Proof. The proof of (7) is similar to that of the well-known formula $\Delta \phi=$ $-m H$ and Formula (8) is an immediate consequence of (7).

Proof of Proposition 2.1. Doing a translation if necessary, we can assume that the center of mass of $\phi$ is at the origin; that is $\int_{M} \phi^{i} d v_{g}=0$ for all $i \leq n$. From the variational characterization of $\lambda_{1}(M)$, we have for any $i$

$$
\lambda_{1}(M) \int_{M}\left(\phi^{i}\right)^{2} d v_{g} \leq \int_{M}\left|d \phi^{i}\right|^{2} d v_{g}
$$

and if the equality holds, then each $\phi^{i}$ is an eigenfunction of the Laplacian. From the above inequality and by applying Lemma 2.3 and using a CauchySchwartz inequality, we obtain the following inequalities

$$
\begin{aligned}
\lambda_{1}(M)\left(\int_{M} \operatorname{tr}(T) d v_{g}\right)^{2} & =\lambda_{1}(M)\left(\int_{M}\left\langle H_{T}, \phi\right\rangle d v_{g}\right)^{2} \\
& \leq \lambda_{1}(M)\left(\int_{M}\left|H_{T}\right|^{2} d v_{g}\right)\left(\int_{M}|\phi|^{2} d v_{g}\right) \\
& \leq\left(\int_{M}\left|H_{T}\right|^{2} d v_{g}\right)\left(\int_{M} \sum_{i}\left|d \phi^{i}\right|^{2} d v_{g}\right) \\
& =m V(M)\left(\int_{M}\left|H_{T}\right|^{2} d v_{g}\right) .
\end{aligned}
$$

This proves the inequality (5) of Proposition 2.1. 
Equality case. If (5) is an equality, then inequalities in (10) are equalities too. But since $\left|H_{T}\right|$ doesn't vanish identically on $M$, we deduce that

$$
\lambda_{1}(M) \sum_{i} \int_{M}\left(\phi^{i}\right)^{2} d v_{g}=\sum_{i} \int_{M}\left|d \phi^{i}\right|^{2} d v_{g}
$$

this implies with (9) that the functions $\phi_{i}$ are eigenfunctions of $\lambda_{1}(M)$. Hence by Takahashi's theorem ([19], Theorem 3) we deduce that $\phi$ is a minimal immersion of $\left(M^{m}, g\right)$ into a hypersphere of radius $\sqrt{m / \lambda_{1}(M)}$.

Proof of Theorem 2.1. The desired inequality can't be deduced from Theorem 1.1, but it will be a consequence of the generalized inequality (5) of Proposition 2.1. In fact, let $T_{r}$ be the $(0,2)$-tensors associated to the second fundamental form $B$ of $\phi$ and let $i$ be the canonical embedding of $\mathbb{S}^{n}$ in $\mathbb{R}^{n+1}$. Then, as before the normal vector field $H_{T_{r}}^{\prime}$ associated to the second fundamental form $B^{\prime}$ of the isometric immersion $i \circ \phi$ is given at $x \in M$ by

$$
H_{T_{r}}^{\prime}=\sum_{1 \leq i, j \leq n} T_{r}\left(e_{i}, e_{j}\right) B^{\prime}\left(e_{i}, e_{j}\right)
$$

where $\left(e_{i}\right)_{1 \leq i \leq m}$ is an orthonormal basis of the tangent space of $M$ at $x$. Now, it follows from (5) that

$$
\lambda_{1}(M)\left(\int_{M} \operatorname{tr}\left(T_{r}\right) d v_{g}\right)^{2} \leq m V(M)\left(\int_{M}\left|H_{T_{r}}^{\prime}\right|^{2} d v_{g}\right)
$$

now, it is easy to see that $B^{\prime}=B-g \phi$ and then $H_{T_{r}}^{\prime}=H_{T_{r}}-\operatorname{tr}\left(T_{r}\right) \phi$. This gives us

$$
\left|H_{T_{r}}^{\prime}\right|^{2}=\left|H_{T_{r}}\right|^{2}+\operatorname{tr}\left(T_{r}\right)^{2}
$$

therefore, reporting this last relation in (11) we obtain

$$
\lambda_{1}(M)\left(\int_{M} \operatorname{tr}\left(T_{r}\right) d v_{g}\right)^{2} \leq m V(M) \int_{M}\left(\left|H_{T_{r}}\right|^{2}+\operatorname{tr}\left(T_{r}\right)^{2}\right) d v_{g} .
$$

Now the inequalities of Theorem 2.1 follow by using Lemma 2.2 which gives us $\left|H_{T_{r}}\right|=k(r)\left|H_{r+1}\right|$ and $\operatorname{tr}\left(T_{r}\right)=k(r) H_{r}$, where $k(r)=(m-r)\left(\begin{array}{c}m \\ r\end{array}\right)$.

Equality case. If we assume that $H_{r}$ doesn't vanish identically, then it is also the case for $H_{T_{r}}^{\prime}$ and we can deduce as in the previous proof, that if equality holds then $M$ is minimally immersed in a geodesic hypersphere of $\mathbb{R}^{n+1}$ with radius less or equal to 1 . If the radius is equal to 1 , then $M$ is minimally immersed in $\mathbb{S}^{n}$ if not $M$ is minimally immersed in a geodesic hypersphere of $\mathbb{S}^{n}$.

Conversely, if $m=n-1$ and if $\phi(M)$ is a geodesic hypersphere of $\mathbb{S}^{n}$, then $\lambda_{1}(M)=(n-1)\left(H_{1}^{2}+1\right)$. On the other hand $H_{r}=H_{1}^{r}$, and inequality (4) becomes an equality. 
These results are a consequence of a Hsiung-Minkowski formulae for submanifolds of $\mathbb{R}^{n}$ or $\mathbb{S}^{n}$. For submanifolds of the hyperbolic space, such a formulae exists but doesn't allow us to generalize these theorems in this case. However, using a different approach, we can obtain a partial result for hypersurfaces of $\mathbb{H}^{n+1}$.

Theorem 2.2. Let $\left(M^{n}, g\right)$ be a compact, orientable $n$-dimensional Riemannian manifold isometrically immersed by $\phi$ into $\mathbb{H}^{n+1}$. Let $r \in\{0, \ldots$, $n-2\}$. If $H_{r}$ is a positive constant and if $\phi$ is convex (i.e., its second fundamental form is semi definite), then we have

$$
\lambda_{1}(M) V(M) H_{r}^{2} \leq n \int_{M}\left(H_{r+1}^{2}-H_{r}^{2}\right) d v_{g}
$$

Moreover, the equality holds if and only if $\phi$ immerses $M$ as a geodesic hypersphere in $\mathbb{H}^{n+1}$.

Proof. Here, $\left(M^{n}, g\right)$ is isometrically immersed in $\mathbb{H}^{n+1}$ and we assume it to be oriented by a unit normal field $\nu$. Therefore as noticed before, the $r$ th mean curvatures will be considered as scalar quantities (see (2)) defined over $M$. In a recent paper, using the fact that any space form $N^{n+1}(\kappa)$ is conformally embedded in $\mathbb{S}^{n+1}$, we establish a relation between $r$-th mean curvatures and the conformal factor $([\mathbf{9}])$. We recall this result in the case which we are interested in, that is when $\kappa=-1$. Let $\Pi$ be a conformal embedding of $\left(\mathbb{H}^{n+1}, \operatorname{can}_{\mathbb{H}}\right)$ into $\left(\mathbb{S}^{n+1}, \operatorname{can}_{\mathbb{S}}\right)$ and let $f$ be the function defined on $\mathbb{H}^{n+1}$ such that $\Pi^{\star} c a n_{\mathbb{S}}=e^{f} c a n_{\mathbb{H}}$. Then we have for any integer $r \in\{0, \ldots, n-1\}$ (see Proposition 3.1 of $[\mathbf{9}]$ )

$$
\begin{aligned}
& H_{r+1}^{2}-H_{r}^{2} \\
& =\left(H_{r+1}-F H_{r}\right)^{2}+e^{f \circ \phi} H_{r}^{2}+\frac{1}{4}\left|\nabla^{M}(f \circ \phi)\right|^{2} H_{r}^{2} \\
& \quad-\frac{1}{2 k(r)} g\left(T_{r} \nabla^{M}(f \circ \phi), \nabla^{M}(f \circ \phi)\right) H_{r}-\frac{1}{k(r)} H_{r} L_{r}(f \circ \phi)
\end{aligned}
$$

where $L_{r}=L_{T_{r}}, F=(1 / 2) \operatorname{can}_{\mathbb{H}}\left(\nabla^{\mathbb{H}^{n+1}} f, \nu\right) \circ \phi, \nabla^{\mathbb{H}^{n+1}}$ and $\nabla^{M}$ denote respectively the gradient of $\mathbb{H}^{n+1}$ and $M$. Furthermore, we have shown (see the proof of Theorem 1.1 of [9]) that for any integer $r \in\{0, \ldots, n-2\}$ and under the assumption of the convexity of $\phi$

$$
\frac{1}{4}\left|\nabla^{M}(f \circ \phi)\right|^{2} H_{r}^{2}-\frac{1}{2 k(r)} g\left(T_{r} \nabla^{M}(f \circ \phi), \nabla^{M}(f \circ \phi)\right) H_{r} \geq 0 .
$$

Since $L_{r}$ is selfadjoint and $H_{r}$ constant, we deduce from (14) and (15) that

$$
\int_{M}\left(H_{r+1}^{2}-H_{r}^{2}\right) d v_{g} \geq \int_{M}\left(H_{r+1}-F H_{r}\right)^{2} d v_{g}+H_{r}^{2} \int_{M} e^{f \circ \phi} d v_{g} .
$$


Now, if we put $X=\Pi \circ \phi$ and if we denote by $X^{i}$ its component functions in $\mathbb{R}^{n+2}$, we have

$$
\sum_{i \leq n+2}\left|d X^{i}\right|^{2}=n e^{f \circ \phi}
$$

Composing $\Pi$ with a conformal diffeomorphism of $\left(\mathbb{S}^{n+1}\right.$, can $)$ if necessary, we can assume that $\int_{M} X^{i} d v_{g}=0([4])$, and thus

$$
\begin{aligned}
& \int_{M}\left(H_{r+1}^{2}-H_{r}^{2}\right) d v_{g} \\
& \geq \int_{M}\left(H_{r+1}-F H_{r}\right)^{2} d v_{g}+\frac{H_{r}^{2}}{n} \int_{M} \sum_{i \leq n+2}\left|d X^{i}\right|^{2} d v_{g} \\
& \geq \frac{H_{r}^{2}}{n} \lambda_{1}(M) \int_{M} \sum_{i \leq n+2}\left(X^{i}\right)^{2} d v_{g}=\frac{H_{r}^{2}}{n} \lambda_{1}(M) V(M) .
\end{aligned}
$$

This proves the inequality in Theorem 2.2.

Equality case. If $\left(M^{n}, g\right)$ is immersed as a geodesic sphere, then $\lambda_{1}(M)=$ $n\left(H_{1}^{2}-1\right)$. Now, since $H_{r}=H_{1}^{r}$, the inequality in Theorem 2.2 becomes an equality. Conversely, assume that (13) is an equality, then all inequalities in (16) are equalities. Thus, $X^{i}$ are eigenfunctions of the Laplacian associated to $\lambda_{1}(M)$ and it follows that

$$
n e^{f \circ \phi}=\sum_{i \leq n+2}\left|d X^{i}\right|^{2}=-\frac{1}{2} \sum_{i \leq n+2} \Delta\left(X^{i}\right)^{2}+\sum_{i \leq n+2} X^{i} \Delta X^{i}=\lambda_{1}(M)
$$

and we deduce that $f \circ \phi$ is constant on $M$. Furthermore, the equality in (16) and Equation (14) imply successively that

$$
\frac{H_{r+1}}{H_{r}}=F
$$

and

$$
e^{f \circ \phi}=\frac{H_{r+1}^{2}}{H_{r}^{2}}-1 .
$$

Now, considering (14) for $r=0$, we have

$$
H_{1}^{2}-1=H_{1}^{2}-2 H_{1} F+F^{2}+e^{f \circ \phi} .
$$

Finally, reporting (17) and (18) in this last equality, we get

$$
H_{r} H_{1}-H_{r+1}=0 .
$$

It is well-known that this implies that $M$ is totally umbilic and thus $\phi(M)$ is a geodesic sphere $([\mathbf{2}])$. 
In the sequel, since the codimension of the orientable manifold $\left(M^{n}, g\right)$ is 1 , we consider $r$-th mean curvatures as scalar quantities (see (2)) defined on $M$. As a straightforward consequences of Theorems 1.1, 2.1 and 2.2 we have the following corollaries:

Corollary 2.1. Let $\left(M^{n}, g\right)$ be a compact, connected orientable n-dimensional Riemannian manifold isometrically immersed by $\phi$ in $\mathbb{R}^{n+1}$. Let $r \in$ $\{1, \ldots, n\}$. If $H_{r}$ is a positive constant, then we have

$$
\lambda_{1}(M) \leq n H_{r}^{2 / r} .
$$

Moreover, we get equality if and only if $\phi$ immerses $\left(M^{n}, g\right)$ as a hypersphere in $\mathbb{R}^{n+1}$.

For hypersurfaces of $\mathbb{S}^{n+1}$, we obtain:

Corollary 2.2. Let $\left(M^{n}, g\right)$ be a compact, connected orientable n-dimensional Riemannian manifold isometrically immersed by $\phi$ in an open hemisphere of $\mathbb{S}^{n+1}$. Let $r \in\{1, \ldots, n-1\}$. If $H_{r+1}>0$ and if $H_{r}$ is a positive constant, then we have

$$
\lambda_{1}(M) \leq n\left(H_{r}^{2 / r}+1\right) .
$$

Moreover, we get equality if and only if $\phi$ immerses $\left(M^{n}, g\right)$ as a hypersphere in $\mathbb{S}^{n+1}$.

And for hypersurfaces of $\mathbb{H}^{n+1}$, we have:

Corollary 2.3. Let $\left(M^{n}, g\right)$ be a compact, connected orientable n-dimensional Riemannian manifold isometrically immersed by $\phi$ in $\mathbb{H}^{n+1}$. For any integer $r \in\{1, \ldots, n-1\}$, if $H_{r}$ is a positive constant and if $\phi$ is convex (i.e., $B$ is semi definite), then we have

$$
\lambda_{1}(M) \leq n\left(H_{r}^{2 / r}-1\right) .
$$

Moreover, we get equality if and only if $\phi$ immerses $\left(M^{n}, g\right)$ as a hypersphere in $\mathbb{H}^{n+1}$.

These corollaries are an immediate consequence of the Maclaurin inequalities which we recall (see for instance [13] and [14]). Let $\phi$ be an isometric immersion of a Riemannian manifold $\left(M^{n}, g\right)$ into a simply connected space form $N^{n+1}(\kappa)\left(\kappa=0,1\right.$ or -1 respectively for $\mathbb{R}^{n+1}, \mathbb{S}^{n+1}$ or $\left.\mathbb{H}^{n+1}\right)$. If for all integer $j \in\{1, \ldots, k\}$, we have $H_{j}>0$ then

$$
H_{k}^{1 / k} \leq H_{j}^{1 / j}
$$

with equality at umbilic points. Moreover, we know that if for an integer $k$, we have:

1. $H_{k}>0$ and $\phi$ is a convex immersion (i.e., $B$ is semi definite), then $H_{j}>0$, for any $j \in\{1, \ldots, k\}([\mathbf{2 0}])$. 
2. $H_{k}>0$ and for $\kappa=1, \phi(M)$ lies in an open hemisphere, then $H_{j}>0$, for any $j \in\{1, \ldots, k\}([5])$.

Note that the Maclaurin inequalities and Property 1 are still valid for hypersurfaces of any ambiant space.

Another approach allows us to obtain a different upper bounds for $\lambda_{1}(M)$ of hypersurfaces of $\mathbb{R}^{n+1}$. Indeed, we have:

Theorem 2.3. Let $\left(M^{n}, g\right)$ be a compact, orientable $n$-dimensional Riemannian manifold isometrically immersed by $\phi$ in $\mathbb{R}^{n+1}$. If for $r \in\{0, \ldots$, $n-2\}$, we have $H_{r+2}>0$, then

$$
\lambda_{1}(M) \int_{M} H_{r} d v_{g} \leq n V(M) \sup _{M} H_{r+2} .
$$

Moreover, equality holds if and only if $\phi$ immerses $\left(M^{n}, g\right)$ as a hypersphere in $\mathbb{R}^{n+1}$.

Proof. From (8), we have

$$
\begin{aligned}
\frac{1}{2}|\phi| L_{r}|\phi|^{2} & =-\left\langle\phi, H_{T_{r}}\right\rangle|\phi|-\operatorname{tr}\left(T_{r}\right)|\phi| \\
& =-k(r)\left(H_{r+1}\langle\phi, \nu\rangle|\phi|+H_{r}|\phi|\right) \\
& \leq k(r)\left(\left|H_{r+1}\right||\phi|^{2}-H_{r}|\phi|\right)
\end{aligned}
$$

hence

$$
\int_{M}|\phi| T_{r}\left(\nabla^{M}|\phi|, \nabla^{M}|\phi|\right) d v_{g} \leq k(r) \int_{M}\left(\left|H_{r+1}\right||\phi|^{2}-H_{r}|\phi|\right) d v_{g} .
$$

Now, in [5] (Proposition 3.2), Barbosa and Colares show that if $H_{r+1}>0$, then $T_{k}$ is a definite positive $(0,2)$-tensor for any $k \in\{1, \ldots, r\}$. Furthermore, we have in particular that $H_{r}>0$. Consequently, we deduce from (19) and the fact that $T_{r}$ is positive, that

$$
\int_{M} H_{r}|\phi| d v_{g} \leq \int_{M} H_{r+1}|\phi|^{2} d v_{g}
$$

and finally from (8) and the above estimate, we obtain

$$
\begin{aligned}
& \lambda_{1}(M) k(r) \int_{M} H_{r} d v_{g} \\
& =\lambda_{1}(M) \int_{M} \operatorname{tr}\left(T_{r}\right) d v_{g} \\
& =-\lambda_{1}(M) \int_{M}\left\langle H_{T_{r}}, \phi\right\rangle d v_{g}
\end{aligned}
$$




$$
\begin{aligned}
& \leq k(r) \lambda_{1}(M) \int_{M} H_{r+1}|\phi| d v_{g} \leq k(r) \lambda_{1}(M) \int_{M} H_{r+2}|\phi|^{2} d v_{g} \\
& \leq k(r) \lambda_{1}(M) \sup _{M} H_{r+2} \int_{M}|\phi|^{2} d v_{g} \leq k(r) \sup _{M} H_{r+2} \int_{M} \sum_{i}\left|d \phi_{i}\right|^{2} d v_{g} \\
& =n k(r) V(M) \sup _{M} H_{r+2} .
\end{aligned}
$$

This completes the proof of Theorem 2.3. Furthermore, it follows from (19) that equality holds if and only if $\phi(M)$ is contained in a geodesic sphere of $\mathbb{R}^{n+1}$.

\section{Upper bounds of $\lambda_{1}(M)$ in terms of scalar curvature.}

First, we deduce from the previous corollaries an unified estimate of $\lambda_{1}(M)$ in terms of the scalar curvature $S$ for hypersurfaces immersed in a space form $N^{n+1}(\kappa)\left(\kappa=0,1\right.$ or -1 respectively for $\mathbb{R}^{n+1}, \mathbb{S}^{n+1}$ and $\left.\mathbb{H}^{n+1}\right)$. Indeed, we have:

Corollary 3.1. Let $\left(M^{n}, g\right)$ be a compact, orientable $n$-dimensional Riemannian manifold isometrically immersed in a simply connected space form $N^{n+1}(\kappa)$. Assume that:

1. If $\kappa=0, r \in\{2, \ldots, n\}$ and $H_{r}$ is a positive constant;

2. if $\kappa=1, r \in\{2, \ldots, n-1\}, \phi(M)$ is contained in an open hemisphere of $\mathbb{S}^{n+1}, H_{r+1}>0$ and $H_{r}$ is a constant;

3. if $\kappa=-1, r \in\{2, \ldots, n-2\}, \phi$ is convex and $H_{r}$ is a positive constant. Then $S>0$, and we have

$$
\lambda_{1}(M) \leq \frac{\inf _{M} S}{n-1} .
$$

Moreover, equality holds if and only if $\phi$ immerses $\left(M^{n}, g\right)$ as a geodesic sphere.

Remark 3.1. If $\left(M^{n}, g\right)$ is an Einstein manifold $(n \geq 3)$ with positive scalar curvature, then the Lichnerowicz-Obata $([\mathbf{1 2}])$ estimate for $\lambda_{1}(M)$ gives us: $\lambda_{1}(M) \geq S /(n-1)$, equality holding only for the spheres. Now, if $\left(M^{n}, g\right)$ is an Einstein manifold of positive scalar curvature isometrically immersed in $\mathbb{R}^{n+1}, H_{2}$ is a positive constant and we deduce from Corollary 3.1 , that $\phi(M)$ is a geodesic sphere. This is another way to prove that the spheres are the only hypersurfaces of $\mathbb{R}^{n+1}$ which are endowed with an Einstein structure of positive scalar curvature (see for instance Theorem 5.3 p. 36 of [11]). We can obtain similar results for the other space forms. Recall that, more generally, Fialkow in [8] proved that geodesic spheres are the only compact Einstein hypersurfaces of positive scalar curvature immersed in a space form $N^{n+1}(\kappa)$. Recall also that A. Montiel and A. Ros in [14] have shown that geodesic spheres are the only compact hypersurfaces of constant 
scalar curvature embedded in $N^{n+1}(\kappa)$ (with the additionaly hypothesis " $\phi(M)$ contained in a hemisphere" for the spherical case $\kappa=1$ ).

Another consequence concerns the Yamabe problem. Indeed, note that T. Aubin ([4]) shows that if $g$ is a Yamabe metric of positive scalar curvature on a compact manifold $\left(M^{n}, g\right)(n \geq 3)$, then $\lambda_{1}(M) \geq S /(n-1)$. Then from our Corollary 3.1, we deduce the following:

Corollary 3.2. If $\left(M^{n}, g\right)$ is a compact hypersurface of positive scalar curvature immersed in $\mathbb{R}^{n+1}$ and if $g$ is a Yamabe metric (i.e., minimizes the Yamabe functional in its conformal class) then $\left(M^{n}, g\right)$ is a standard sphere.

Proof of Corollary 3.1. This corollary follows from Corollaries 2.1, 2.2 and 2.3 , in the case $r=2$. Under the assumptions of these corollaries and by using the Maclaurin inequalities about $r$-th mean curvatures, we obtain

$$
\lambda_{1}(M) \leq n\left(H_{r}^{2 / r}+\kappa\right) \leq n\left(H_{2}+\kappa\right)
$$

and equality holds if and only if $\phi$ immerses $\left(M^{n}, g\right)$ as a geodesic sphere. Now, let $\left(e_{i}\right)_{1 \leq i \leq n}$ be a $g$-orthonormal basis which diagonalizes the second fundamental form $b$ (i.e., $\left.b\left(e_{i}, e_{j}\right)=\left\langle B\left(e_{i}, e_{j}\right), \nu\right\rangle=\mu_{i} \delta_{i j}\right)$. From the Gauss equation, we have

$$
S=\kappa n(n-1)+\sum_{i \neq j} \mu_{i} \mu_{j}=n(n-1)\left(\kappa+H_{2}\right)
$$

and reporting this relation in (20), we obtain the desired inequality.

As an immediate consequence of Theorem 2.3, we have $\lambda_{1}(M) \leq$ $\sup _{M} S /(n-1)$, by applying the inequality for $r=0$. The techniques used in this theorem don't allow us to extend it to hypersurfaces of $\mathbb{S}^{n+1}$ and $\mathbb{H}^{n+1}$. But, by a different method inspired by Heintze's work $([\mathbf{1 0}])$, we can prove:

Theorem 3.1. Let $\left(M^{n}, g\right)$ be a compact, orientable $n$-dimensional Riemannian manifold isometrically immersed in a simply connected space form $N^{n+1}(\kappa)\left(\kappa=0,1\right.$ or -1 respectively for $\mathbb{R}^{n+1}, \mathbb{S}^{n+1}$ or $\left.\mathbb{H}^{n+1}\right)$ and assume in addition that for $\kappa=1, \phi(M)$ lies in a geodesic ball of radius $\pi / 4$. If $S>n(n-1) \kappa$ then we have

$$
\lambda_{1}(M) \leq \frac{\sup _{M} S}{n-1}
$$

and equality holds if and only if $\phi$ immerses $M$ as a geodesic sphere.

Before giving the proof of Theorem 3.1, we need to give some preliminary results. Let $p_{0} \in N^{n+1}(\kappa)$ and $\exp _{p_{0}}$ the exponential map at this point. We denote $\left(x_{i}\right)_{1 \leq i \leq n+1}$ the normal coordinates of $N^{n+1}(\kappa)$ centered at $p_{0}$ and for all $x \in N^{n+1}(\kappa)$, we set $r(x)=d\left(p_{0}, x\right)$, the geodesic distance between 
$p_{0}$ and $x$ on $N^{n+1}(\kappa)$. Assume in the case $\kappa=1$, that $\phi(M)$ lies in an open hemisphere.

Let $s_{\kappa}$ and $c_{\kappa}$ be the functions defined by

$$
s_{\kappa}(r)=\left\{\begin{array}{ll}
\sin r & \text { if } \kappa=1 \\
r & \text { if } \kappa=0 \\
\sinh r & \text { if } \kappa=-1
\end{array} \text { and } \quad c_{\kappa}(r)= \begin{cases}\cos r & \text { if } \kappa=1 \\
1 & \text { if } \kappa=0 \\
\cosh r & \text { if } \kappa=-1\end{cases}\right.
$$

Note that $c_{\kappa}^{2}+\kappa s_{\kappa}^{2}=1$ and $s_{\kappa}^{\prime}=c_{\kappa}$ and $c_{\kappa}^{\prime}=-s_{\kappa}$.

In the sequel, we denote respectively by $\nabla^{M}$ and $\nabla^{N}$ the gradient associated to $g$ and to the canonical metric of $N^{n+1}(\kappa)$ denoted by $h$. Then, if we put $X=s_{\kappa}(r) \nabla^{N} r$, it is easy to see that the normal coordinates of $X$ are $\left(\frac{s_{\kappa}(r)}{r} x_{i}\right)_{1 \leq i \leq n+1}$. Furthermore, the tangential and the normal projection of a vector field $Y$ respectively on the tangent bundle and the normal bundle to $\phi(M)$ will be denoted by $Y^{T}$ and $Y^{\perp}$.

We recall two lemmas shown by Heintze $([\mathbf{1 0}])$ :

Lemma 3.1. At any $x \in M$, we have

$$
\sum_{1 \leq i \leq n+1} g_{x}\left(\nabla^{M}\left(\frac{s_{\kappa}(r)}{r} x_{i}\right), \nabla^{M}\left(\frac{s_{\kappa} k(r)}{r} x_{i}\right)\right)=n-\kappa g_{x}\left(X^{T}, X^{T}\right)
$$

Lemma 3.2. The vector field $X=s_{\kappa}(r) \nabla^{N} r$ satisfies

$$
\kappa \int_{M}\left|X^{T}\right|^{2} d v_{g}=n \int_{M} c_{\kappa}^{2} d v_{g}-n \int_{M}|H| s_{\kappa} c_{\kappa} d v_{g}
$$

Now, we need the following inequality for the proof of Theorem 3.1:

Lemma 3.3. For all symmetric free divergence definite positive (0,2)-tensor $T$, we have

$$
\operatorname{tr}(T) c_{\kappa} \leq s_{\kappa}\left|H_{T}\right|+\operatorname{div}_{M}\left(T^{\sharp} X^{T}\right)
$$

and if $T$ is the identity, then equality holds.

Proof of Lemma 3.3. Since $T^{\sharp}$ is a positive symmetric $(1,1)$-tensor, we can define a natural positive symmetric $(1,1)$-tensor $\sqrt{T^{\sharp}}$ such that $\sqrt{T^{\sharp}} \circ \sqrt{T^{\sharp}}=$ $T^{\sharp}$.

Now let $\left(e_{i}\right)_{1 \leq i \leq n}$ be an orthonormal frame at $x$, such that $\sqrt{T^{\sharp}} e_{n}$ lies in the direction of $\nabla^{M} r$ and let $e_{n}^{*}$ be a unit vector orthogonal to $\nabla^{N} r$ in order 
to have: $\sqrt{T^{\sharp}} e_{n}=\lambda \nabla^{N} r+\mu e_{n}^{*}$. Then at $x$, we have

$$
\begin{aligned}
\operatorname{div}_{M}\left(T^{\sharp} X^{T}\right) & =\sum_{1 \leq i \leq n} g_{x}\left(\nabla_{e_{i}}^{M}\left(T^{\sharp} X^{T}\right), e_{i}\right)=\sum_{1 \leq i \leq n} h_{x}\left(\nabla_{e_{i}}^{N} X^{T}, T^{\sharp} e_{i}\right) \\
& =\sum_{1 \leq i \leq n} h_{x}\left(\nabla_{e_{i}}^{N} X, T^{\sharp} e_{i}\right)-\sum_{1 \leq i \leq n} h_{x}\left(\nabla_{e_{i}}^{N} X^{\perp}, T^{\sharp} e_{i}\right) \\
& =\sum_{1 \leq i \leq n} h_{x}\left(\nabla_{e_{i}}^{N} X, T^{\sharp} e_{i}\right)+h_{x}\left(X, H_{T}\right) .
\end{aligned}
$$

We need to estimate $\sum_{1 \leq i \leq n} h_{x}\left(\nabla_{e_{i}}^{N} X, T^{\sharp} e_{i}\right)$. We first have

$$
\begin{aligned}
& \sum_{1 \leq i \leq n} h_{x}\left(\nabla_{e_{i}}^{N} X, T^{\sharp} e_{i}\right) \\
= & \sum_{1 \leq i \leq n} h_{x}\left(\nabla_{e_{i}}^{N}\left(s_{\kappa} \nabla^{N} r\right), T^{\sharp} e_{i}\right) \\
= & c_{\kappa} h_{x}\left(\nabla^{N} r, T^{\sharp}\left(\nabla^{N} r\right)^{T}\right)+s_{\kappa} \sum_{1 \leq i \leq n} h_{x}\left(\nabla_{e_{i}}^{N} \nabla^{N} r, T^{\sharp} e_{i}\right) \\
= & c_{\kappa} h_{x}\left(T^{\sharp}\left(\nabla^{N} r\right)^{T},\left(\nabla^{N} r\right)^{T}\right)+s_{\kappa} \sum_{1 \leq i \leq n} h_{x}\left(\nabla_{\sqrt{T^{\sharp}} e_{i}}^{N} \nabla^{N} r, \sqrt{T^{\sharp}} e_{i}\right) .
\end{aligned}
$$

Now, we compute the last term of (24). Using the Jacobi fields of $N^{n+1}(\kappa)$, one can prove that $D^{2} r=\left(c_{\kappa} / s_{\kappa}\right)(h-d r \otimes d r)$ (see for instance [18]). Then, for all orthogonal vector $\xi$ to $\nabla^{N} r$ at $x$, we have the equality

$$
h_{x}\left(\nabla_{\xi}^{N} \nabla^{N} r, \xi\right)=\frac{c_{\kappa}}{s_{\kappa}}|\xi|_{x}^{2} .
$$

Thus

$$
\begin{aligned}
& \sum_{1 \leq i \leq n} h_{x}\left(\nabla_{\sqrt{T^{\sharp}} e_{i}}^{N} \nabla^{N} r, \sqrt{T^{\sharp}} e_{i}\right) \\
= & \sum_{1 \leq i \leq n-1} h_{x}\left(\nabla_{\sqrt{T^{\sharp}} e_{i}}^{N} \nabla^{N} r, \sqrt{T^{\sharp}} e_{i}\right)+h_{x}\left(\nabla_{\sqrt{T^{\sharp}} e_{n}}^{N} \nabla^{N} r, \sqrt{T^{\sharp}} e_{n}\right) \\
= & \frac{c_{\kappa}}{s_{\kappa}} \sum_{1 \leq i \leq n-1}\left|\sqrt{T^{\sharp}} e_{i}\right|_{x}^{2}+\mu^{2} h_{x}\left(\nabla_{e_{n}^{*}}^{N} \nabla^{N} r, e_{n}^{*}\right) \\
= & \frac{c_{\kappa}}{s_{\kappa}} \sum_{1 \leq i \leq n-1}\left|\sqrt{T^{\sharp}} e_{i}\right|_{x}^{2}+\mu^{2} \frac{c_{\kappa}}{s_{\kappa}}
\end{aligned}
$$

and reporting this inequality in (24), we obtain

$$
\begin{aligned}
& \sum_{1 \leq i \leq n} h_{x}\left(\nabla_{e_{i}}^{N} X, T^{\sharp} e_{i}\right) \\
& =c_{\kappa}\left|\sqrt{T^{\sharp}}\left(\nabla^{N} r\right)^{T}\right|_{x}^{2}+c_{\kappa} \sum_{1 \leq i \leq n-1}\left|\sqrt{T^{\sharp}} e_{i}\right|_{x}^{2}+\mu^{2} c_{\kappa}
\end{aligned}
$$


now

$$
\lambda^{2}=h_{x}\left(\sqrt{T^{\sharp}} e_{n}, \nabla^{N} r\right)^{2}=h_{x}\left(e_{n}, \sqrt{T^{\sharp}}\left(\nabla^{N} r\right)^{T}\right)^{2} \leq\left|\sqrt{T^{\sharp}}\left(\nabla^{N} r\right)^{T}\right|_{x}^{2}
$$

and if $T^{\sharp}$ is the identity, this last inequality is in fact an equality. Furthermore, it is easy to verify that

$$
\lambda^{2}+\mu^{2}=\left|\sqrt{T^{\sharp}} e_{n}\right|_{x}^{2} .
$$

Thus, from (25) and these two last facts, we have

$$
\begin{aligned}
\sum_{1 \leq i \leq n} h_{x}\left(\nabla_{e_{i}}^{N} X, T^{\sharp} e_{i}\right) & \geq c_{\kappa}\left(\lambda^{2}+\mu^{2}+\sum_{1 \leq i \leq n-1}\left|\sqrt{T^{\sharp}} e_{i}\right|_{x}^{2}\right) \\
& =\operatorname{tr}(T) c_{\kappa} .
\end{aligned}
$$

Now, we report this last inequality in (23) and we complete the Proof of Lemma 3.3 by noting that $h_{x}\left(X, H_{T}\right) \geq-|X|\left|H_{T}\right|=-s_{\kappa}\left|H_{T}\right|$.

Now, we can give the Proof of Theorem 3.1:

Proof of Theorem 3.1. Let $p_{0} \in N$ and $r(x)=d\left(p_{0}, x\right)$. We will use $\frac{s_{\kappa}(r)}{r} x_{i}$ as test functions in the variational characterization of $\lambda_{1}(M)$ but the mean of these functions must be zero. For this purpose, we use a standard argument used by Chavel and Heintze before ([10] and [6]). Indeed, let $Y$ be the vector field defined by

$$
Y_{q}=\int_{M} \frac{s_{\kappa}(d(q, p))}{d(q, p)} \exp _{q}^{-1}(p) d v_{g}(p) \in T_{q} N .
$$

From the theorem of fixed point of Brouwer, there exists a point $p_{0} \in N$ such that $Y_{p_{0}}=0$ and consequently, for a such $p_{0}$, the mean of $\frac{s_{\kappa}(r)}{r} x_{i}$ will be zero. But for $\kappa=1$, we must assume $\phi(M)$ contained in a ball of radius $\pi / 4$. This guarantees the inclusion of $\phi(M)$ in a ball of center $p_{0}$ (the point $p_{0}$ such that $Y_{p_{0}}=0$ ) with a radius less or equal to $\pi / 2$ (this hypothesis is necessary in the proof of the preceding lemmas). It follows from the variational characterization of $\lambda_{1}(M)$, that

$$
\begin{aligned}
& \lambda_{1}(M) \int_{M} s_{\kappa}^{2}(r) d v_{g} \\
& =\lambda_{1}(M) \int_{M}|X|^{2} d v_{g}=\lambda_{1}(M) \int_{M} \sum_{1 \leq i \leq n+1}\left(\frac{s_{\kappa}(r)}{r} x_{i}\right)^{2} d v_{g} \\
& \leq \int_{M} \sum_{1 \leq i \leq n+1} g\left(\nabla^{M}\left(\frac{s_{\kappa}(r)}{r} x_{i}\right), \nabla^{M}\left(\frac{s_{\kappa}(r)}{r} x_{i}\right)\right) d v_{g}
\end{aligned}
$$


and using Lemmas 3.1 and 3.2, we deduce that

$$
\begin{aligned}
\lambda_{1}(M) \int_{M} s_{\kappa}^{2}(r) d v_{g} & \leq n V(M)-\kappa \int_{M}\left|X^{T}\right|^{2} d v_{g} \\
& \leq n \kappa \int_{M} s_{\kappa}^{2} d v_{g}+n \int_{M}|H| s_{\kappa} c_{\kappa} d v_{g} \\
& =n \kappa \int_{M} s_{\kappa}^{2} d v_{g}+\frac{1}{n-1} \int_{M} \operatorname{tr}\left(T_{1}\right) s_{\kappa} c_{\kappa} d v_{g}
\end{aligned}
$$

now, from Lemma 3.3, we have

$$
\operatorname{tr}\left(T_{1}\right) s_{\kappa} c_{\kappa} \leq s_{\kappa} \operatorname{div}_{M}\left(T_{1}^{\sharp} X^{T}\right)-h\left(X, H_{T_{1}}\right) s_{\kappa}
$$

and reporting this inequality in (26), we obtain

$$
\begin{aligned}
& \lambda_{1}(M) \int_{M} s_{\kappa}^{2} d v_{g} \\
& \leq n \kappa \int_{M} s_{\kappa}^{2} d v_{g}-\frac{1}{n-1} \int_{M} h\left(X, H_{T_{1}}\right) s_{\kappa} d v_{g}+\frac{1}{n-1} \int_{M} s_{\kappa} \operatorname{div}_{M}\left(T_{1}^{\sharp} X^{T}\right) d v_{g} \\
& \leq n \kappa \int_{M} s_{\kappa}^{2} d v_{g}+\frac{1}{n-1} \int_{M}\left|H_{T_{1}}\right| s_{\kappa}^{2} d v_{g}-\int_{M} g\left(\nabla^{M} s_{\kappa}, T_{1}^{\sharp} X^{T}\right) d v_{g} \\
& =n \kappa \int_{M} s_{\kappa}^{2} d v_{g}+n \int_{M} H_{2} s_{\kappa}^{2} d v_{g}-\int_{M} s_{\kappa} c_{\kappa} T_{1}\left(\nabla^{M} r, \nabla^{M} r\right) d v_{g} .
\end{aligned}
$$

Since we assume that $S>n(n-1) \kappa$, it follows from $(21)$, that $H_{2}>0$, and from the same argument used in the proof of Theorem 2.3, $T_{1}$ is a definite positive $(0,2)$-tensor $([5])$. Furthermore $c_{\kappa}$ and $s_{\kappa}$ are positive functions and thus

$$
\lambda_{1}(M) \int_{M} s_{\kappa}^{2} d v_{g} \leq n \int_{M}\left(H_{2}+\kappa\right) s_{\kappa}^{2} d v_{g}=\frac{1}{n-1} \int_{M} S s_{\kappa}^{2} d v_{g}
$$

which gives the inequality of Theorem 3.1. Now, equality in this inequality holds if and only if $T_{1}\left(\nabla^{M} r, \nabla^{M} r\right)=0$. Since $T_{1}$ is definite positive, this is the case if and only if $\phi(M)$ is a geodesic sphere. This concludes the Proof of Theorem 3.1.

Acknowledgements. The author would like to express his gratefulness to his advisor Professor S. Ilias.

\section{References}

[1] A.R. Aithal and G. Santhanam, Sharp upper bound for the first nonzero Neumann eigenvalue for bounded domains in rank-1 symmetric spaces, Trans. Amer. Math. Soc., 348(10) (October 1996), 3955-3965, MR 96m:58252, Zbl 0866.35081.

[2] H. Alencar, M. do Carmo and H. Rosenberg, On the first eigenvalue of the linearized operator of the $r$-th mean curvature of a hypersurface, Ann. Global Anal. Geom., 11 (1993), 387-395, MR 96c:53091, Zbl 0816.53031. 
[3] Erratum to On the first eigenvalue of the linearized operator of the $r$-th mean curvature of a hypersurface, Ann. Global Anal. Geom., 13 (1995), 99-100, MR 96c:53091, Zbl 0822.53035.

[4] T. Aubin, Nonlinear Analysis on Manifolds, Monge-Ampère Equation, Grundlehren Math.Wiss., 252, Springer, 1982, MR 85j:58002, Zbl 0512.53044.

[5] J.L. Barbosa and A.G. Colares, Stability of hypersurfaces with constant $r$-mean curvature, Ann. Global Anal. Geom., 15 (1997), 277-297, MR 98h:53091, Zbl 0891.53044.

[6] I. Chavel, Riemannian Geometry-A Modern Introduction, Cambridge University Press, 1993, MR 95j:53001, Zbl 0810.53001.

[7] A. El Soufi and S. Ilias, Une inegalité du type "Reilly" pour les sous-variétés de l'espace hyperbolique, Comm. Math. Helv., 67 (1992), 167-181, MR 93i:53059, Zbl 0758.53029.

[8] A. Fialkow, Hypersurfaces of a space of constant curvature, Ann. of Math., 39 (1938), 762-785, Zbl 0020.06601.

[9] J.F. Grosjean, A Reilly inequality for some natural elliptic operators on hypersurfaces, Differential Geom. Appl., 13 (2000), 267-276, MR 2001k:53114, Zbl 0977.53052.

[10] E. Heintze, Extrinsic upper bound for $\lambda_{1}$, Math. Ann., 280 (1988), 389-402, MR 89f:53091, Zbl 0628.53044.

[11] S. Kobayashi and K. Nomizu, Foundations of Differential Geometry, 2, Wiley and Sons, New York, 1969, MR 38 \#6501, Zbl 0175.48504.

[12] A. Lichnerowicz, Géométrie des Groupes de Transformation, Dunod, Paris, 1958, MR 23 \#A1329, Zbl 0096.16001.

[13] M. Lin and N.S. Trudinger, On some inequalities for elementary symmetric functions, Bull. Austral. Math. Soc., 50 (1994), 317-326, MR 95i:26036, Zbl 0855.26006.

[14] S. Montiel and A. Ros, Compact hypersurfaces: The Alexandrov theorem for higher order mean curvatures, Differential Geometry, Blaine Lawson and Keti Tonenblat, Pitman Monographs, 52 (1991), 279-297, MR 93h:53062, Zbl 0723.53032.

[15] R. Reilly, Applications of the Hessian operator in a Riemannian manifold, Indiana Univ. Math. J., 26 (1977), 459-472, MR 57 \#13799, Zbl 0391.53019.

[16] _ On the first eigenvalue of the Laplacian for compact submanifolds of Euclidean space, Comment. Math. Helv., 52 (1977), 525-533, MR 58 \#2657, Zbl 0382.53038.

[17] H. Rosenberg, Hypersurfaces of constant curvature in space forms, Bull. Sc. Math., 117 (1993), 211-239, MR 94b:53097, Zbl 0787.53046.

[18] T. Sakai, Riemannian Geometry, A.M.S. translations of Math. Monographs, 149, Amer. Math. Soc., 1996, MR 97f:53001, Zbl 0886.53002.

[19] T. Takahashi, Minimal immersions of Riemannian manifolds, J. Math. Soc. Japan, 18 (1966), 380-385, MR 33 \#6551, Zbl 0145.18601.

[20] R. Walter, Compact hypersurfaces with a constant higher mean curvature function, Math. Ann., 270 (1985), 125-145, MR 86f:53068, Zbl 0536.53054. 
Received December 28, 2000.

Institut Élie Cartan (Mathématiques)

Université Henri POINCARÉ NANCY I

B.P. 239

F-54506 Vandoeuvre-Les-Nancy Cedex, France

E-mail address: grosjean@iecn.u-nancy.fr 